\title{
Experiences with a training program in the use of methods other than seclusion and restraint
} Anna Bjorkdahl

\author{
Address: Department of Clinical Neuroscience, Division of Psychiatry, Karolinska Institute Huddinge, SE14186 Stockholm, Sweden \\ from WPA Thematic Conference. Coercive Treatment in Psychiatry: A Comprehensive Review \\ Dresden, Germany. 6-8 June 2007 \\ Published: 19 December 2007 \\ BMC Psychiatry 2007, 7(SuppI I):S64 doi:10.1 I86/I47I-244X-7-SI-S64
}

This abstract is available from: http://www.biomedcentral.com/I47I-244X/7/SI/S64

(C) 2007 Bjorkdahl; licensee BioMed Central Ltd.

\section{Background}

Prevention of patient violence should be initiated very early in the aggression process. In order to improve the early preventive interventions on a psychiatric intensive care unit (PICU), we introduced the Bröset Violence Checklist (BVC) and structured preventive care plans.

\section{Methods}

The BVC estimates the patient's level of risk for violence during the next 24 hours. In the checklist six behavior items are noted as present or not present: confusion, irritability, boisterousness, verbal threats, physical threats and attacks on objects. On the PICU, a nurse was assigned on each shift to assess the patients. If more than two items were present, interventions to prevent a violent incident had to be initiated. We also developed a standardized list that stated common etiological factors, short-term goals and nursing interventions, especially designed for patients at risk of becoming violent towards others. The nursing interventions covered aspects of patient participation, information, support, general care, environment, observation and coordination. By using relevant parts of the list, an individualized preventive care plan could be in clinical use shortly after the admittance of a new patient. All members of staff were obliged to read the patients' care plans before entering the ward and was expected to carry out the interventions accordingly.

\section{Results and conclusion}

The staff found the BVC easy to learn and use. An evaluation of the predictive capacity of the BVC used on the PICU showed that the risk for violence in a short term perspective could to a high degree be predicted by the nursing staff. Similarly, we found that the standardized list for preventive care plans was experienced as helpful and an often necessary tool. It enabled the nurses to quickly set up care plans also under acute and stressful situations and was considered well suited to be combined with the BVC. 